\title{
Left-sided multifractality in a binary random multiplicative cascade
}

\author{
W. G. Hanan, ${ }^{1}$ J. Gough, ${ }^{1,2}$ and D. M. Heffernan ${ }^{1,3}$ \\ ${ }^{1}$ Department of Mathematical Physics, National University of Ireland Maynooth, Maynooth, County Kildare, Ireland \\ ${ }^{2}$ Department of Mathematics, Statistics and Operational Research, Nottingham Trent University, \\ Burton Street, Nottingham NG1 4BU, England \\ ${ }^{3}$ School of Theoretical Physics, Dublin Institute for Advanced Studies, Dublin 4, Ireland
}

(Received 10 November 1999; revised manuscript received 19 July 2000; published 27 December 2000)

In this paper we study a binary random multiplicative cascade. Specifically, the cascade is used to produce and study left-sided multifractal random measures. Extensive numerical simulations of the random cascade process were undertaken and $f(\alpha)$ spectra obtained and compared with the analytical results. We believe that this model and approach can serve as a simple and fundamental tool in the analysis and understanding of physical systems possessing an underlying multiplicative structure.

DOI: 10.1103/PhysRevE.63.011109

PACS number(s): 05.40.-a, 05.45.Df

\section{INTRODUCTION}

The multiplicative cascade has been the cornerstone on which the theory of multifractal measures [1-6] has been built over the last three decades. In the introduction of standard texts on multifractal theory, the binomial measure [6], constructed via a multiplicative cascade, is often used as a first, simple, lucid example of a self-similar measure and introduces us to such concepts as the Holder exponent $\alpha$ and the functions $\tau(q)$ and $f(\alpha)$. However, the deterministic nature of the model does not reflect the property that in the natural world, measures are created through random processes. Examples of such measures include the energy dissipation in space of a turbulent flow [3-5,7], the growth probability of such kinetic growth processes as colloidal and DLA-like aggregation $[8,9]$ and the rapidity of particles generated by high energy collisions [10].

In this paper the binomial model is generalized by introducing randomness into the construction process. This random model produces statistically self-similar measures. The $f(\alpha)$ function of such measures may display properties which differ markedly from those resulting from a purely deterministic model. As a specific example, we examine numerically a form which produces left-sided multifractal measures (as defined by Mandelbrot et al. [11]). Such measures may be relevant to the study of DLA where the growth probability on the boundary of the cluster exhibit multifractal characteristics which are synonymous with left-sided measures.

The paper begins by first giving a brief introduction of the binomial measure in Sec. II. In Sec. III we generalize this model by treating the multipliers as random variables. In Sec. IV we choose a specific form for the probability density function of this random variable and show that the $f(\alpha)$ function of the resulting measure is left-sided. And finally in Sec. V we investigate how the numerical results for the $f(\alpha)$ compare with the analytical results from Sec. IV.

\section{THE BINOMIAL MEASURE}

The binomial measure is constructed via a multiplicative cascade. First, two positive values $m_{0}$ and $m_{1}$ are chosen such that $m_{0}+m_{1}=1$ (let $\left.m_{0}>m_{1}\right)$. The value of these multipliers is kept constant throughout the construction process.

One begins with the unit interval $I=[0,1]$ with the measure $\mu$ initially uniformly distributed on it. The initial amount of measure existing on the interval $I$ is set to unity, i.e., $\mu(I)=1$. In the first stage $(k=1)$ of construction we fragment this measure in two, placing a fraction $m_{0}$ of the measure uniformly on the interval $I_{0}=\left[0, \frac{1}{2}\right]$ and the remaining fraction $m_{1}=1-m_{0}$ uniformly on $I_{1}=\left[\frac{1}{2}, 1\right]$. We thus have that $\mu\left(I_{0}\right)=m_{0}$ and $\mu\left(I_{1}\right)=m_{1}$.

The measure at the $k=2$ stage is obtained by repeating this procedure separately on the measure $\mu\left(I_{0}\right)$ existing on $I_{0}$ and the measure $\mu\left(I_{1}\right)$ on $I_{1}$. That is we take the measure $\mu\left(I_{0}\right)$ and fragment it in two, placing a fraction $m_{0}$ of it uniformly on the interval $I_{00}=\left[0, \frac{1}{4}\right]$ and the remaining fraction $m_{1}$ uniformly on $I_{01}=\left[\frac{1}{4}, \frac{1}{2}\right]$. Similarly with the measure $\mu\left(I_{1}\right)$ on $I_{1}$.

Thus at the $k=2$ stage, there are $2^{k}=4$ intervals each of length $2^{-k}=\frac{1}{4}$, each holding the measure

$$
\begin{aligned}
& \mu\left(I_{00}\right)=m_{0} m_{0}, \quad \mu\left(I_{01}\right)=m_{0} m_{1}, \\
& \mu\left(I_{10}\right)=m_{1} m_{0}, \quad \mu\left(I_{11}\right)=m_{1} m_{1} .
\end{aligned}
$$

Note that we identify an interval at the $k$ th stage of the cascade uniquely via an address consisting of a string of $k$ digits $\beta_{1} \beta_{2} \ldots \beta_{k}$, where $\beta_{i} \in\{0,1\}$ and $i \in\{1,2, \ldots, k\}$. The address of the interval $\left[n 2^{-k},(n+1) 2^{-k}\right]$, where $n$ $\in\left\{0,1,2, \ldots, 2^{k}-1\right\}$ is simply given by the binary expansion of $n$ expressed to $k$ digits.

Following this iterative procedure, the measure at any stage $k$ is easily generated with the binomial measure defined in the limit $k \rightarrow \infty$. It is easily observed that

$$
\mu\left(I_{\beta_{1} \beta_{2} \ldots \beta_{k}}\right)=m_{\beta_{1}} m_{\beta_{2}} \ldots m_{\beta_{k}} .
$$

Defining the coarse Holder exponent $\alpha$ of an interval to be

$$
\alpha=\frac{\log \mu\left(I_{\beta_{1} \beta_{2} \ldots \beta_{k}}\right)}{\log \left|I_{\beta_{1} \beta_{2} \ldots \beta_{k}}\right|}=\frac{\log \Pi_{i=1}^{k} m_{\beta_{i}}}{\log 2^{-k}}=\frac{1}{k} \sum_{i=1}^{k} v_{i},
$$


where $v_{i}=-\log _{2} m_{\beta_{i}}$, one finds that asymptotically as $k$ $\rightarrow \infty$ the number of intervals $N_{k}(\alpha) d \alpha$ at the $k$ th level in the cascade with a coarse Holder exponent between $\alpha$ and $\alpha$ $+d \alpha$ scales as

$$
N_{k}(\alpha) \sim\left(2^{-k}\right)^{-f(\alpha)}=\epsilon^{-f(\alpha)} .
$$

The function $f(\alpha)$ is thus a measure of the rate of increase of the number of intervals characterized by the exponent $\alpha$ as $k$ increases, or conversely as the length scale $\epsilon$ $=2^{-k}$ decreases.

Analytically the simplest approach to finding $f(\alpha)$ is via the method of moments. This involves the calculation of the function $\tau(q)$ defined as

$$
\tau(q)=\lim _{\epsilon \rightarrow 0} \frac{\log \Sigma_{i} \mu_{i}^{q}}{\log \epsilon}
$$

where the summation is taken over all $2^{k}$ intervals existing at the $k$ th stage of the cascade and $\mu_{i}$ denotes the measure of the $i$ th interval.

For the binomial measure we have that at stage $k$

$$
\sum_{i} \mu_{i}^{q}=\left(m_{0}^{q}+m_{1}^{q}\right)^{k}
$$

and it is subsequently easily obtained that $\tau(q)$ is independent of $k$ and is given by

$$
\tau(q)=-\log _{2}\left(m_{0}^{q}+m_{1}^{q}\right) .
$$

The $f(\alpha)$ and $\tau(q)$ functions can be shown to be related via the Legendre transform

$$
f(\alpha)=\min _{q}\{\alpha q-\tau(q)\}
$$

or more specifically, assuming the function $\tau(q)$ to be continuous and differentiable everywhere as in the present example, by Ref. [1]:

$$
\begin{gathered}
f(\alpha)=\alpha q-\tau(q), \\
\alpha=\frac{d \tau(q)}{d q} .
\end{gathered}
$$

From Eq. (7) and Eq. (9) we thus obtain the function

$$
\begin{aligned}
f(\alpha)= & -\left(\frac{\alpha_{\max }-\alpha}{\alpha_{\max }-\alpha_{\min }}\right) \log _{2}\left(\frac{\alpha_{\max }-\alpha}{\alpha_{\max }-\alpha_{\min }}\right) \\
& -\left(\frac{\alpha-\alpha_{\min }}{\alpha_{\max }-\alpha_{\min }}\right) \log _{2}\left(\frac{\alpha-\alpha_{\min }}{\alpha_{\max }-\alpha_{\min }}\right)
\end{aligned}
$$

defined on the domain $\left[\alpha_{\min }, \alpha_{\max }\right]$ where $\alpha_{\min }=-\log _{2} m_{0}$ and $\alpha_{\max }=-\log _{2} m_{1}$. Note that the Legendre transform (9) implies that $f(\alpha)$ possesses a unique maximum at $q=0$. Thus, the value of $\alpha$ at which $f(\alpha)$ is a maximum is usually denoted by $\alpha_{0}$. For the binomial measure

$$
\alpha_{0}=\frac{1}{2}\left(v_{0}+v_{1}\right) .
$$
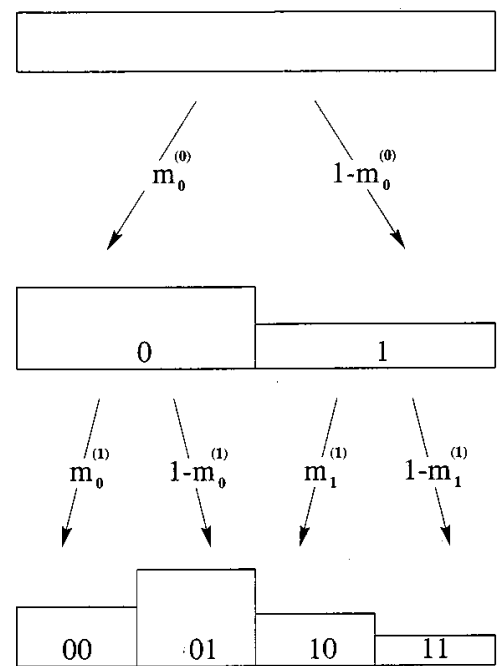

FIG. 1. Diagram showing the splitting of the measure at successive stages in the construction of the cascade. Note that $m_{j}^{(i)}$ is simply the $j$ th sample value of the random variable $\mathbf{M}_{0}$ used to fragment the measure onto the $(i+1)$ th stage. At each stage $2^{i}$ such sample values are required (thus $j \in\left\{0,1,2, \ldots, 2^{i}-1\right\}$ ).

Intervals possessing this value $\alpha_{0}$ will by definition dominate all others in the limit $k \rightarrow \infty$, i.e., the number of intervals with $\alpha=\alpha_{0}$ divided by the total number of intervals at any level $k$ tends to one as $k \rightarrow \infty$.

\section{THE RANDOM BINARY MULTIPLICATIVE CASCADE}

The binomial model was introduced as a means of constructing self-similar measures. As previously mentioned, once the value of the multiplier $m_{0}$ is chosen, the model and the measure it produces is entirely deterministic. The determinism shall now be eliminated by making the model random. One constructs the measure as before but now with one important modification.

Whenever it comes to redistribute the measure $\mu_{\beta_{1} \beta_{2} \ldots \beta_{k}}$ onto its subintervals $I_{\beta_{1} \beta_{2} \ldots \beta_{k} 0}$ and $I_{\beta_{1} \beta_{2} \ldots \beta_{k} 1}$ the values of the multipliers $m_{0}$ and $m_{1}$ are now chosen randomly under the constraint $m_{0}+m_{1}=1$ so as to once again ensure conservation of the measure. As we are attempting to construct measures which resemble those found in the natural world, when generating the measure we choose $2^{k}$ random pairs $\left(m_{0}, m_{1}\right)$ at each stage $k$ in the cascade (and not one pair as is usually done). These random values provide the multipliers with which to fragment the measure and subsequently generate the measure at the $(k+1)$ level (see Fig. 1).

In the language of probability theory, vectors $\mathbf{M}$ $=\left(\mathbf{M}_{0}, \mathbf{M}_{1}\right)$ are chosen randomly from the sample space

$$
\Omega=\left\{\left(\mathbf{M}_{0}, \mathbf{M}_{1}\right), \mathbf{M}_{j} \geqslant 0, \sum_{j=0}^{1} \mathbf{M}_{j}=1\right\} \subset \mathbf{R}^{2}
$$

according to some predetermined probability measure $P$. That is, the probability of choosing a vector $\mathbf{M}$ which lies in a subset $A \in \Omega$ is 


$$
P^{\mathbf{M}}(A)=\int_{\mathbf{M} \in A} \rho(m) d m
$$

where $\rho(m)$ is some probability density function. The sample values of the component variables $\mathbf{M}_{0}$ and $\mathbf{M}_{1}$ provides one with the multipliers with which to fragment the measure. Note that due to the constraint, $\rho(m)$ is a function of only one of the component variables.

The subsequent random measure produced via this procedure is not exactly self-similar as is the case with the binomial measure but is statistically self-similar in the sense that the probability density function $\rho(m)$ through which the random values of the multipliers are chosen is kept constant throughout the entire construction process.

We now apply the ideas and concepts encountered in the deterministic model to the random model. We wish to evaluate the $f(\alpha)$ function with which we can characterize the scaling properties of the resultant measure. Again we shall do this via the method of moments. As the $\Sigma \mu^{q}$ is now a random quantity dependent upon the particular realization of the cascade obtained, we calculate its average over the ensemble of all possible cascades. Specifically we could take the annealed and quenched average and define the functions

$$
\begin{gathered}
\tau_{A}(q) \underset{\substack{\epsilon \rightarrow 0 \\
\epsilon \rightarrow 0}}{=} \frac{\log \left\langle\left\langle\sum \mu^{q}\right\rangle\right\rangle}{\log \epsilon}, \\
\tau_{Q}(q) \underset{\substack{\epsilon \rightarrow 0 \\
\epsilon \rightarrow 0}}{\langle} \frac{\left\langle\left\langle\log \Sigma \mu^{q}\right\rangle\right\rangle}{\log \epsilon},
\end{gathered}
$$

where the brackets $\langle\langle\rangle\rangle$ denote the average over all members of the stochastic ensemble. From an experimental viewpoint, the function $\tau_{Q}(q)$ corresponding to a quenched average is usually calculated due to its greater statistical robustness. Normally $\tau_{A}(q) \neq \tau_{Q}(q)$ except for a few special values of $q$ such as $q=0,1$. For now, we shall concentrate on the evaluation of the annealed exponents $\tau_{A}(q)$ as they prove to be analytically tractable.

Choosing an interval at the $k$ th stage of construction with address $\beta=\beta_{1} \beta_{2} \ldots \beta_{k}$ where again $\beta_{i} \in\{0,1\}$ we have that the measure in this interval is given by

$$
\mu\left(I_{\beta}\right)=\prod_{i=1}^{k} \mathbf{M}_{\beta_{i}} .
$$

That is, the measure on any interval on the $k$ th level of the cascade is the product of $k$ independent random variables. As a consequence we have that

$$
\begin{aligned}
\left\langle\left\langle\sum \mu^{q}\right\rangle\right\rangle & =\left\{E\left[\mathbf{M}_{0}^{q}+\mathbf{M}_{1}^{q}\right]\right\}^{k} \\
& \Rightarrow \tau_{A}(q)=-\log _{2} E\left[\mathbf{M}_{0}^{q}+\mathbf{M}_{1}^{q}\right],
\end{aligned}
$$

where we have

$$
E\left[\mathbf{M}_{0}^{q}+\mathbf{M}_{1}^{q}\right]=\int_{0}^{1}\left\{m_{0}{ }^{q}+\left(1-m_{0}\right)^{q}\right\} \rho\left(m_{0}\right) d m_{0} .
$$

The coarse Holder exponent $\alpha$ of an interval on the $k$ th level is given by

$$
\alpha=\frac{\log \mu\left(I_{\beta}\right)}{\log \left|I_{\beta}\right|}=\frac{1}{k} \sum_{i=1}^{k} \mathbf{V}_{\beta_{i}}
$$

where $\mathbf{V}_{\beta_{i}}=-\log _{2} \mathbf{M}_{\beta_{i}}$. In the limit $k \rightarrow \infty$ the law of large numbers ensures that

$$
\operatorname{Pr}\left\{\lim _{k \rightarrow \infty} \frac{1}{k} \sum_{i=1}^{k} \mathbf{V}_{\beta_{i}}=E\left[\frac{1}{2}\left(\mathbf{V}_{0}+\mathbf{V}_{1}\right)\right]\right\}=1
$$

Consequently we have by definition

$$
\alpha_{0}=E\left[\frac{1}{2}\left(\mathbf{V}_{0}+\mathbf{V}_{1}\right)\right]
$$

Working in terms of the random vector $\mathbf{V}=\left(\mathbf{V}_{0}, \mathbf{V}_{1}\right)$, where as stated above $\mathbf{V}_{0}=-\log _{2} \mathbf{M}_{0}$ and $\mathbf{V}_{1}=-\log _{2} \mathbf{M}_{1}$ we may rewrite Eq. (18) in terms of $v_{0}$ and putting into Eq. (17) we obtain

$$
\tau_{A}(q)=-\log _{2}\left\{\int_{0}^{\infty}\left(e^{-q v_{0} \ln 2}+\left(1-e^{-v_{0} \ln 2}\right)^{q}\right) \widetilde{\rho}\left(v_{0}\right) d v_{0}\right\}
$$

where $\widetilde{\rho}\left(v_{0}\right)$ is the probability density function of the random vector $\mathbf{V}$. It is a simple matter to show that the probability density functions $\rho\left(m_{0}\right)$ and $\widetilde{\rho}\left(v_{0}\right)$ are related by

$$
\widetilde{\rho}\left(v_{0}\right)=2^{-v_{0}} \ln (2) \rho\left(2^{-v_{0}}\right) \text {. }
$$

Once the form of $\rho\left(m_{0}\right)$ or equivalently $\widetilde{\rho}\left(v_{0}\right)$ is specified, the scaling properties of the resultant measure produced by our binary random model can be characterized by the evaluation of $\tau_{A}(q)$ from Eq. (22).

\section{LEFT-SIDED MULTIFRACTALITY}

Let us now choose the following heavy-tailed power law form for $\tilde{\rho}\left(v_{0}\right)$

$$
\widetilde{\rho}\left(v_{0}\right)=\frac{\lambda}{\left(1+v_{0}\right)^{1+\lambda}}, \quad 0 \leqslant v_{0}<\infty
$$

where the parameter $\lambda>0$.

For $q<0$ it is easily obtained from Eq. (22) that $\tau_{A}(q)$ $=-\infty$. Applying the Legendre transform (8) we obtain the degenerate result $f(\alpha)=-\tau_{A}(0)=1$ for $\alpha>\alpha_{0}$. Consequently, as we shall see, it is only the left-hand side of the $f(\alpha)$ function which is defined. The measure generated through using Eq. (24) is thus known as a left-sided multifractal measure.

Putting Eq. (24) into Eq. (22) we find that unfortunately no closed form expression exists for $\tau_{A}(q)$ for $q \geqslant 0$. However we are able to obtain an expression in the regime 0 $<q \ll 1$. The details of the calculation may be found in the appendix. Thus for $0<q \ll 1$ we obtain 


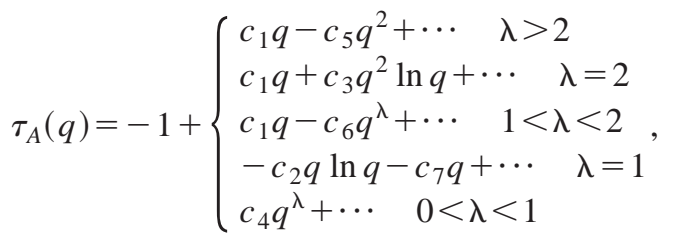

where the constants $c_{1}, c_{2}, \ldots, c_{7}$ are functions of $\lambda$. It should be stated that this result has previously been obtained by Mandelbrot et al. in their original work on left-sided multifractality [11]. However the model which they investigated was entirely deterministic in nature, consisting of a deterministic multiplicative cascade with an infinite base.

Evaluating $\alpha_{0}$ from Eq. (21) gives us that

$$
\begin{aligned}
& \alpha_{0}(\lambda) \\
& \quad=\left\{\begin{array}{l}
\infty, \quad \lambda \leqslant 1 \\
\frac{1}{2(\lambda-1)}-\frac{1}{2} \int_{0}^{\infty} \log _{2}\left(1-e^{-v \ln 2}\right) \widetilde{\rho}_{0}(v) d v, \quad \lambda>1
\end{array}\right.
\end{aligned}
$$

and we discover that the constant $c_{1}=\alpha_{0}(\lambda)$ with $\lambda>1$. Applying the Legendre transform (9) on the expression for $\tau_{A}(q)$ above we obtain the following results for the form of $f(\alpha)$ about $\alpha_{0}$

$$
f(\alpha) \simeq 1-\left\{\begin{array}{l}
b_{1}\left[\alpha_{0}(\lambda)-\alpha\right]^{\gamma}, \quad \lambda>1, \alpha \nearrow \alpha_{0}(\lambda) \\
b_{2} e^{-c^{\prime} \alpha}, \quad \lambda=1, \alpha \rightarrow \infty \\
b_{3} \alpha^{\kappa}, \quad 0<\lambda<1, \alpha \rightarrow \infty
\end{array}\right.
$$

where $b_{1}, b_{2}$ and $b_{3}$ are positive constants which depend on $\lambda, c^{\prime}=2, \kappa=\lambda /(\lambda-1)$ and $\gamma=\max \{\lambda /(\lambda-1), 2\}$.

The phase transition at the critical point $q=0$ in the $\tau_{A}(q)$ function [resulting in the left-sided nature of the $f(\alpha)]$ is a consequence of a breakdown in scaling [12-14]. In multifractal theory, it is assumed that the measure $\mu$ in a box of size $\epsilon$ scales as

$$
\mu \underset{\epsilon \rightarrow 0}{\sim} \epsilon^{\alpha},
$$

where $\alpha$ is the Holder exponent. The existence of points which violate this ensatz may lead to the appearance of phase transitions in the $\tau_{A}(q)$ function such as the one seen above.

For the specific form (24), using a heuristic argument similar to Hentschel's [8], one may estimate that the minimal measure $\mu_{\min }$ in existence at any resolution $\epsilon$ scales as

$$
\mu_{\min }(\boldsymbol{\epsilon}) \sim \exp \left[-c \epsilon^{-1 / \lambda}\right],
$$

where $c$ is a positive constant. This has a stretched exponential form and not the power law form of Eq. (28). For negative $q$ the existence of this anomalous scaling form dominates the quantity $\Sigma \mu^{q}$ in Eq. (14a) as $\epsilon \rightarrow 0$, thus resulting in the nonfiniteness of $\tau_{A}(q)$ for $q<0$.

\section{NUMERICAL RESULTS}

Using the heavy-tailed power law form proposed for $\widetilde{\rho}\left(v_{0}\right)$ in the previous section, simulations of the cascade process were performed on a computer for the values of $\lambda$ $=0.5,1.0,1.2,1.5,2.0,5.0$. The simulation was terminated once the computer had constructed the measure to the $k$ $=24$ th stage. For each value of $\lambda, 1000$ simulations were performed to obtain reliable statistics. The algorithm used to simulate the cascade process requires little computer memory though the run time increases exponentially with $k$.

Having generated these measures, the primary objective was to evaluate their $f(\alpha)$ functions, concentrating particularly on the maximum of the $f(\alpha)$ spectra where analytical results have been obtained. Accordingly, the method of moments was employed for a range of $q$ values about zero $(-0.005 \leqslant q \leqslant 0.014)$. For each $\lambda$ value, both the annealed and quenched average of the quantity $\Sigma \mu^{q}$ was taken over the 1000 cascades.

Plots of $\log \left\langle\left\langle\Sigma \mu^{q}\right\rangle\right\rangle$ vs $\log \epsilon$ and $\left\langle\left\langle\log \Sigma \mu^{q}\right\rangle\right\rangle$ vs $\log \epsilon$ were produced and the expected linear scaling region was found (for $q>0$ ) at low values of $\epsilon$ (over $\left.\epsilon \approx 2^{-12} \rightarrow 2^{-24}\right)$. The functions $\tau_{A}(q)$ and $\tau_{Q}(q)$ were calculated from the slopes of these scaling regions (14) and subsequently, $f(\alpha)$ functions were evaluated via the Legendre transform (9). For each value of $\lambda$, it was found that (for the range of $q$ values examined) the $f(\alpha)$ functions corresponding to the annealed and quenched averages were equal to within numerical accuracy.

The full $f(\alpha)$ functions for these measures were also estimated numerically via a technique known as the histogram method [6]. Briefly, one calculates the coarse holder exponent $\alpha(=\ln \mu / \ln \epsilon)$ of each box on the $k$ th level of the cascade and estimates the frequency distribution $N_{k}(\alpha)$ of these $\alpha$ values using a fixed bin size $\Delta \alpha$ for this histogram plot. Having obtained this plot one rescales the $y$ axis by taking

$$
-\frac{\ln \left(N_{k}(\alpha) / \Delta \alpha\right)}{\ln \epsilon}=f_{k}(\alpha)
$$

One thus obtains a series of plots for $f_{k}(\alpha)$, one for each value of $k$. [For the moment we shall postpone till later any discussion on precisely how one evaluates an average $f_{k}(\alpha)$ plot over the 1000 realisations of the cascade.] One expects these plots of $f_{k}(\alpha)$ to collapse onto a common curve as $k$ $\rightarrow \infty$ if the measure is indeed multifractal. This common curve is the $f(\alpha)$ function of the measure.

Looking at Fig. 2 one can see that the $f_{k}(\alpha)$ curves display behavior synonymous with left-sided multifractalitycollapse of the curves at low values of $\alpha$ and a lack of such convergence at larger values. Clearly significant finite size effects are present at these larger values and are problematic to the estimation of a complete $f(\alpha)$ function.

In order to check for convergence in these plots and estimate an $f(\alpha)$ function for comparison to the analytical results, the following procedure was performed. Taking the set of $f_{k}(\alpha)$ curves belonging to a particular $\lambda$ value, an $\alpha$ value was chosen and a graph of $f_{k}(\alpha)$ vs $k$ was plotted. If the $f_{k}(\alpha)$ 's are converging at this value of $\alpha$ one expects to see 

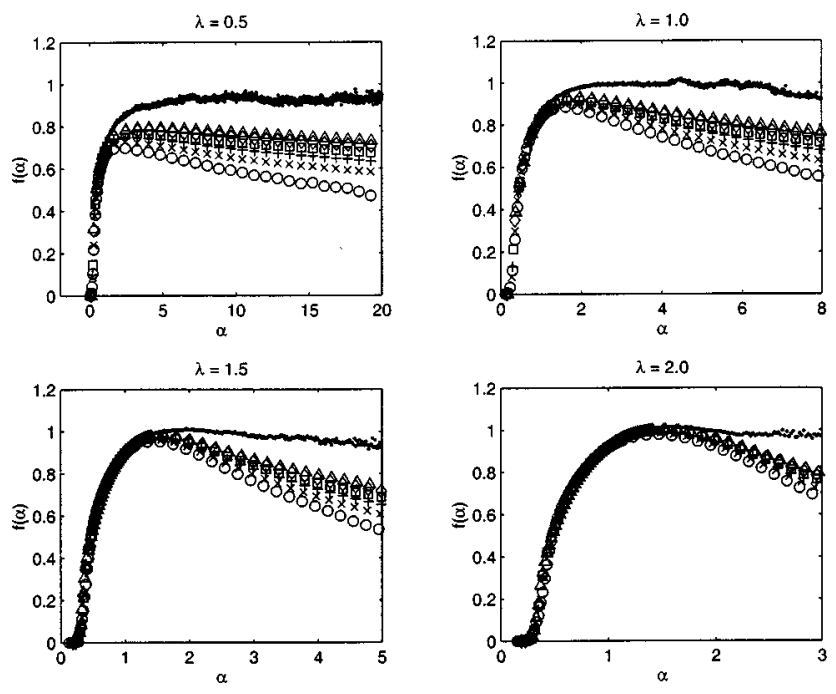

FIG. 2. The $f_{k}(\alpha)$ curves for four different values of $\lambda$ corresponding to the values $k=14(\bigcirc), 16(\times), 18(+)$, $20(\square), 22(\diamond), 24(\triangle)$. The extrapolated $f(\alpha)$ function $(\cdot)$ is also shown.

this plot level off with increasing $k$. Such behavior was indeed observed as illustrated in Fig. 3. In order to predict the asymptotic value of $f_{k}(\alpha)$ from such a plot, a curve of the following form was fitted to the data points in the $f_{k}(\alpha)$ vs $k$ graph

$$
f_{k}(\alpha)=A-\Gamma_{B, C}(k),
$$

where $A, B$, and $C$ are the three positive fitting parameters and $\Gamma_{B, C}(k)$ is some function which goes to zero as $k \rightarrow \infty$. Note that this fit was performed only over the larger values of $k$ where convergence was evident. Various forms for $\Gamma_{B, C}(k)$ were chosen and tested on the data plots. These included exponential $\left(\sim e^{-C k}\right)$, inverse logarithmic $(\sim 1 /$ ln $C k)$ and power law $\left(\sim k^{-C}\right)$ decay with $k$. The testing involved performing a three parameter $(A, B, C)$ fit on the

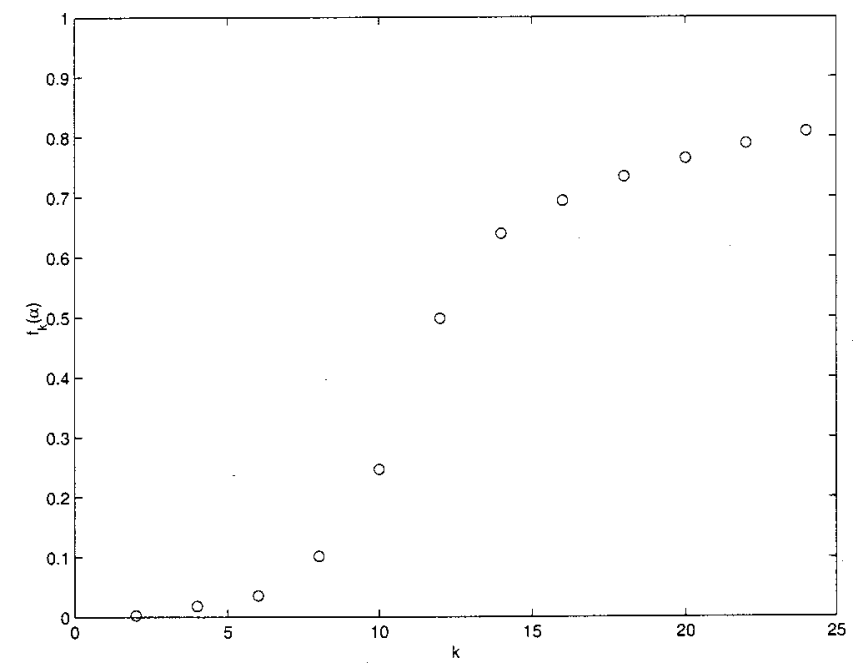

FIG. 3. Sample plot of $f_{k}(\alpha)$ vs $k$ with $\alpha=5.99$, taken from the $f_{k}(\alpha)$ curves for $\lambda=1.0$. Note the beginnings of convergence of this curve to some asymptotic value at the larger values of $k$.
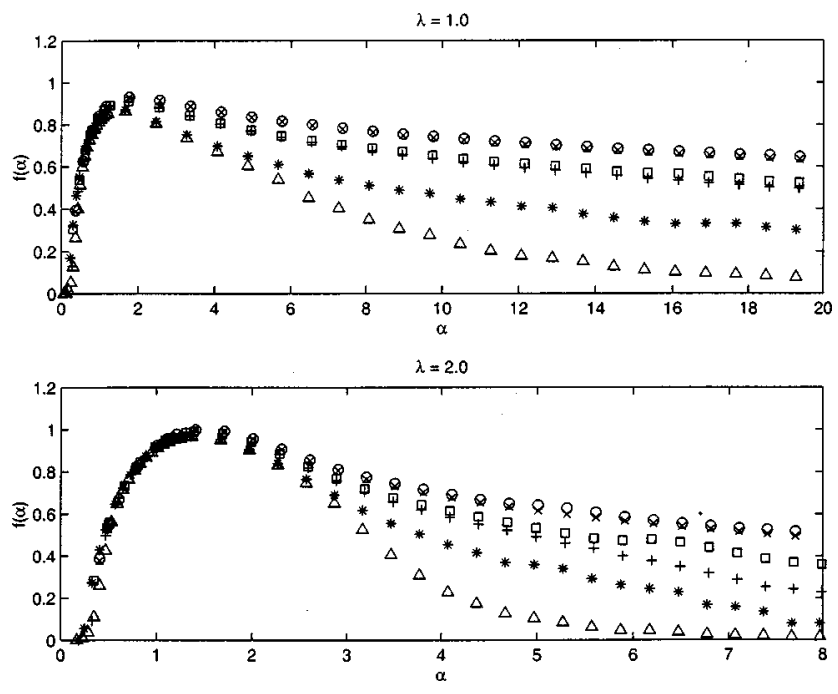

FIG. 4. Plot of annealed $f_{k}(\alpha)$ curves for $k=12(*), k=18$ (square) and $k=24(\bigcirc)$ for $\lambda=1$ and $\lambda=2$. For comparison purposes the quenched $f_{k}(\alpha)$ curves for $k=12(\triangle), k=18(+)$ and $k=24(\times)$ are also shown. Note that as $k$ increases the annealed and quenched $f_{k}(\alpha)$ curves appear to converge.

$f_{k}(\alpha)$ vs $k$ data plots using the standard LevenbergMarquardt method [15]. The fitting procedure thus estimates values for $A, B$, and $C$. (The value returned for $A$ is the only parameter of interest as this gives the asymptotic value of the plot.) The form of $\Gamma_{B, C}(k)$ eventually chosen was the power law

$$
\Gamma_{B, C}(k)=B k^{-C}
$$

The criterion used in choosing the above form of $\Gamma_{B, C}(k)$ over the alternative forms was simply that it returned values for $f\left(\alpha_{0}\right)$, the maximum of the $f(\alpha)$ which were in close agreement with the expected value $\left(f\left(\alpha_{0}\right)=1\right)$.

For most values of $\alpha$ the above form gave a good fit to the data. Automating this curve-fitting procedure, the asymptotic value of $f_{k}(\alpha)$ was estimated for every $\alpha$ value available and subsequently an extrapolated $f(\alpha)$ function was obtained. The extrapolated $f(\alpha)$ functions for the different $\lambda$ values are shown in Fig. 2. This simple procedure for estimating the $f(\alpha)$ function proved to be remarkably successful. [One can clearly see the flattening of the $f(\alpha)$ to the degenerate result $f(\alpha)=1$ in the graphs for $\lambda>1$.]

At this point one should note that when implementing the histogram method, one must again average over the 1000 realizations of the cascade. As such, for each value of $k$, one may take either an average over the frequency distributions $N_{k}(\alpha)$ and then rescale according to Eq. (30) or take an average over the $f_{k}(\alpha)$ plots themselves (the former is an annealed average, the latter a quenched average). Both averages were performed numerically and it was found that as $k$ increased, the $f_{k}(\alpha)$ plots of both averages appear to converge to the same curve. (See Fig. 4.) Consequently, as found with the method of moments there appears to be little difference numerically between the annealed and quenched versions of the $f(\alpha)$. 
TABLE I. Numerical results of three parameter nonlinear fit performed on the $f(\alpha)$ spectra about their maxima. Results for both the extrapolated $f(\alpha)$ of the histogram method and the $f(\alpha)$ calculated via the method of moments are given. The form of the fitting curve is given in the table. Note that only two of the three parameters are quoted.

\begin{tabular}{|c|c|c|c|c|}
\hline$\lambda$ & $f(\alpha)$ & $\begin{array}{c}\text { Theoretical } \\
\text { values }\end{array}$ & $\begin{array}{l}\text { Histogram } \\
\text { method }\end{array}$ & $\begin{array}{l}\text { Method of } \\
\text { moments }\end{array}$ \\
\hline 0.5 & $A-b_{1} \alpha^{\kappa}$ & $\begin{array}{c}A=1.0 \\
\kappa=-1.0\end{array}$ & $\begin{array}{c}A=0.967 \pm 0.007 \\
\kappa=-1.015 \pm 0.099\end{array}$ & $\begin{array}{c}A=1.000 \pm 0.001 \\
\kappa=-0.940 \pm 0.018\end{array}$ \\
\hline 1.0 & $A-b_{2} e^{-c^{\prime} \alpha}$ & $\begin{array}{l}A=1.0 \\
c^{\prime}=2.0\end{array}$ & $\begin{array}{l}A=0.997 \pm 0.001 \\
c^{\prime}=2.096 \pm 0.054\end{array}$ & $\begin{array}{l}A=1.000 \pm 0.001 \\
c^{\prime}=1.581 \pm 0.029\end{array}$ \\
\hline 1.2 & $A-b_{3}\left[\alpha_{0}-\alpha\right]^{\gamma}$ & $\begin{aligned} A & =1.0 \\
\gamma & =6.0 \\
\alpha_{0} & \approx 4.30\end{aligned}$ & $\begin{array}{c}A=1.002 \pm 0.001 \\
\gamma=6.073 \pm 0.328 \\
\alpha_{0} \approx 3.37\end{array}$ & $\begin{array}{c}A=1.000 \pm 0.001 \\
\gamma=2.770 \pm 0.125 \\
\alpha_{0} \approx 3.13\end{array}$ \\
\hline 1.5 & $A-b_{3}\left[\alpha_{0}-\alpha\right]^{\gamma}$ & $\begin{aligned} A & =1.0 \\
\gamma & =3.0 \\
\alpha_{0} & \approx 2.26\end{aligned}$ & $\begin{array}{c}A=1.008 \pm 0.001 \\
\gamma=2.979 \pm 0.196 \\
\alpha_{0} \approx 1.95\end{array}$ & $\begin{array}{c}A=1.000 \pm 0.001 \\
\gamma=2.550 \pm 0.071 \\
\alpha_{0} \approx 2.18\end{array}$ \\
\hline 2.0 & $A-b_{3}\left[\alpha_{0}-\alpha\right]^{\gamma}$ & $\begin{aligned} A & =1.0 \\
\gamma & =2.0 \\
\alpha_{0} & \approx 1.70\end{aligned}$ & $\begin{array}{c}A=1.014 \pm 0.001 \\
\gamma=2.028 \pm 0.130 \\
\alpha_{0} \approx 1.57\end{array}$ & $\begin{array}{c}A=1.000 \pm 0.001 \\
\gamma=2.031 \pm 0.330 \\
\alpha_{0} \approx 1.70\end{array}$ \\
\hline 5.0 & $A-b_{3}\left[\alpha_{0}-\alpha\right]^{\gamma}$ & $\begin{aligned} A & =1.0 \\
\gamma & =2.0 \\
\alpha_{0} & \approx 1.77\end{aligned}$ & $\begin{array}{c}A=1.016 \pm 0.001 \\
\gamma=2.037 \pm 0.089 \\
\alpha_{0} \approx 1.73\end{array}$ & $\begin{array}{c}A=1.000 \pm 0.001 \\
\gamma=2.039 \pm 0.005 \\
\alpha_{0} \approx 1.77\end{array}$ \\
\hline
\end{tabular}

Having obtained estimates for the $f(\alpha)$ function via the histogram method and the method of moments for each $\lambda$ value, a curve of the expected form, as predicted by Eq. (27), was fitted (again using the Levenberg-Marquardt algorithm) about the maximum of the $f(\alpha)$ and values for the exponents $\gamma, c^{\prime}$ and $\kappa$ were calculated and compared to the theoretical values. The results are summarized in Table I. Note that in this table there is no distinction made between quenched or annealed averages as for both methods numerically, there is little if any difference.

The error bars given are merely statistical in nature. For example, with reference to the results obtained from the histogram method, the error bars do not take into account any systematic error which results from the extrapolation procedure performed on the $f_{k}(\alpha)$ curves. As a result the theoretical value for $A$ (see Table I), the maximum of the $f(\alpha)$, consistently lies outside the range of the error bars given.

The values obtained for the critical exponents $\kappa, c^{\prime}$ and $\gamma$ via the histogram method are surprisingly accurate [considering the simplicity of the method used in generating an extrapolated $f(\alpha)$ function]. The numerical value of $\alpha_{0}$ quoted for the histogram method was obtained from simply identifying the maximum of the extrapolated $f(\alpha)$. As $\lambda$ approaches 1 from above, it became increasingly difficult to ascertain an exact value of $\alpha_{0}$ as the region close to the maximum $\left(\alpha<\alpha_{0}\right)$ of the $f(\alpha)$ becomes stretched and flattens out. This can be problematic as the value obtained for $\gamma$ via a numerical fit proves very sensitive to the value of $\alpha_{0}$ used.
The method of moments provides us with an extremely accurate numerical estimate of $A$ for all $\lambda$. However, one can see from the table that as $\lambda \rightarrow 1$ from above, the values of $\alpha_{0}$ and $\gamma$ deviate increasingly from the analytical values. Primarily, this is a result of the central difference method used in evaluating the local slope of the $\tau(q)$ function. Accurate evaluation of the slope of $\tau(q)$ is critical as, can be seen from Eq. (9), it provides the values of $\alpha$ and in turn the values of $f(\alpha)$. From the form of $\tau_{A}(q)$ given in Eq. (25) one can calculate that for a fixed $q$ in the positive neighborhood of $q=0$, the truncation error of the estimated slope at $q$ increases as $\lambda \rightarrow 1$. Thus it is not surprising that the results become worse as $\lambda$ reduces to one. In our numerical work we chose twenty $q$ values spaced out equally over intervals of fixed size $\Delta q=0.001$ in the range $-0.005 \leqslant q \leqslant 0.014$. Choosing smaller interval sizes (though more computationally intensive) leads to improved results.

Though the method of moments does not suffer to the same degree from the debilitating finite size effects observed in the histogram method it does suffer from other problems. For $q<0$, the smallest values of the measure $\mu$ dominate the quantity $\Sigma \mu^{q}$. As the parameter $\lambda$ decreases to zero, smaller values of $\mu$ become more likely to appear in the cascade as the probability distribution function $\rho\left(m_{0}\right)$ becomes increasingly weighted towards $m_{0}=0$. This is problematic for negative $q$ as if small enough values of $\mu$ exist then the quantity $\Sigma \mu^{q}$ becomes extremely large and exceeds the range of floating point numbers which the computer can represent. If this occurs, the computer effectively treats the quantity $\Sigma \mu^{q}$ 
as infinite and one is unable to generate the necessary $\log \Sigma \mu^{q}$ vs $\log \epsilon$ graphs required to implement the method.

For the same reason, there may also be a significant error in the estimation of $\Sigma \mu^{q}$ for $q \geqslant 0$. During the generation of the cascade, values of $\mu$ may be produced which are less than the smallest floating point number representable on the computer. The computer treats such values as zero. This unfortunately means that these values of $\mu$ do not contribute to the quantity $\Sigma \mu^{q}$ as they should, leading to the aforementioned error. This error increases with decreasing $q$ and would become more significant at lower values of $\lambda$ for the reason outlined briefly in the previous paragraph.

\section{DISCUSSION AND SUMMARY}

In this paper a simple random binary multiplicative model is introduced for the production of statistically self-similar measures. The model is simple and easily implemented algorithmically. As one is free to choose any (normalizable) form for the probability density function $\rho\left(m_{0}\right)$ or equivalently $\widetilde{\rho}\left(v_{0}\right)$, the $f(\alpha)$ function characterizing the resultant random measures is expected to display the wide and diverse range of behavior associated with such measures: negative values for $f(\alpha)$, the presence of phase transitions and nonfiniteness of the interval $\left[\alpha_{\min }, \alpha_{\max }\right]$.

It therefore should not be unexpected that random selfsimilar measures found in the natural world should exhibit such behavior. Taking the growth probability of DLA as an example, the $f(\alpha)$ function displays finite size effects that may be indicative of a left-sided multifractal measure. How does the simple random binary model in this paper relate to the problem of DLA? Well, the measure (growth probability) along some interval on the boundary of the cluster may be viewed as the result of some underlying multiplicative process $[8,16]$ as embodied by Eq. (15). The sample values of the random variable $\mathbf{M}$ in this case would correspond to the probability of a random walker successfully negotiating each "stage" (the series of bottlenecks and channels) in its journey towards the particular boundary interval of interest. These sample values could take on any value in the interval $0 \leqslant m \leqslant 1$, the left-hand inequality being a necessary condition for left-sided multifractality. The exact form of $\rho(m)$ would need to be obtained before coming to any conclusion on the left-sided issue (assuming of course that a problem as seemingly complex as DLA could be described by something as simplistic as the random binary model) [8].

Motivated by the possible connection between DLA and left-sided multifractality, the simple form (24) for $\widetilde{\rho}\left(v_{0}\right)$ was chosen as a specific example for study. The random measures produced by such a model turn out to be equivalent to the family of exactly self-similar left-sided nonrandom fractal measures investigated previously by Mandelbrot et al. [11].

Significant finite size effects were encountered on implementing the histogram method in the attempt to numerically calculate the $f(\alpha)$ function. Confronted with this problem a simple method for estimating the $f(\alpha)$ from its finite-size approximations was implemented. The $f(\alpha)$ functions subsequently attained were compared to the analytical predictions and were found to agree closely.

For $q>0$ the method of moments was found not to suffer from any significant finite size effects and subsequently the numerical results obtained in the positive neighborhood of $q=0$ were, on the whole, in close agreement with the analytical values. [Decreasing the length $\Delta q$ between successive points on the $\tau(q)$ graph should improve the results further.] However, due to the inherent limit of a computer in representing large numbers, the method proved unworkable for large negative $q$ values.

The model presented is binary in nature. However the arguments and theory outlined in this paper are easily extended to a model of any base $b$ and not just $b=2$.

\section{ACKNOWLEDGMENT}

We wish to acknowledge the support of Enterprise Ireland (the Irish funding agency for science and technology).

\section{APPENDIX: DERIVATION OF $\tau_{A}(q)$ IN THE REGIME $0<q \ll 1$ FOR POWER LAW TAILED PROBABILITY FUNCTION $\widetilde{\rho}\left(v_{0}\right)$}

We have that

$$
\tau_{A}(q)=-\log _{2} E\left[\mathbf{M}_{0}^{q}+\mathbf{M}_{1}^{q}\right] .
$$

Making the change of variable to $v_{0}=-\log _{2} m_{0}$

$$
\begin{aligned}
E\left[\mathbf{M}_{0}^{q}+\left(1-\mathbf{M}_{0}\right)^{q}\right]= & \int_{0}^{\infty}\left(e^{-q v_{0} \ln 2}+\left(1-e^{-v_{0} \ln 2}\right)^{q}\right) \\
& \times \widetilde{\rho}\left(v_{0}\right) d v_{0},
\end{aligned}
$$

where we have chosen for $\lambda>0$, the following form for $\widetilde{\rho}\left(v_{0}\right)$

$$
\widetilde{\rho}\left(v_{0}\right)=\left\{\begin{array}{l}
0, \quad 0<v_{0}<v_{c} \\
\frac{\lambda c}{\left(1+v_{0}\right)^{1+\lambda}}, \quad v_{c} \leqslant v_{0}<\infty
\end{array}\right.
$$

with $c=\left(1+v_{c}\right)^{\lambda}$ to ensure normalization.

We wish to evaluate Eq. (A1) in the regime $0<q \ll 1$ for the function $\widetilde{\rho}\left(v_{0}\right)$ above. Let us begin by writing Eq. (A2) as

$$
E\left[\mathbf{M}_{0}^{q}+\left(1-\mathbf{M}_{0}\right)^{q}\right]=I_{0}+I_{1},
$$

where

$$
\begin{gathered}
I_{0}=\lambda c \int_{v_{c}}^{\infty} \frac{e^{-q v_{0} \ln 2}}{\left(1+v_{0}\right)^{1+\lambda}} d v_{0}, \\
I_{1}=\lambda c \int_{v_{c}}^{\infty} \frac{\left(1-e^{-v_{0} \ln 2}\right)^{q}}{\left(1+v_{0}\right)^{1+\lambda}} d v_{0} .
\end{gathered}
$$

For a given $\lambda$ we have that $(n-1)<\lambda \leqslant n$ where $n$ is some integer greater than zero. Integrating $I_{0}$ by parts $n$ times gives 


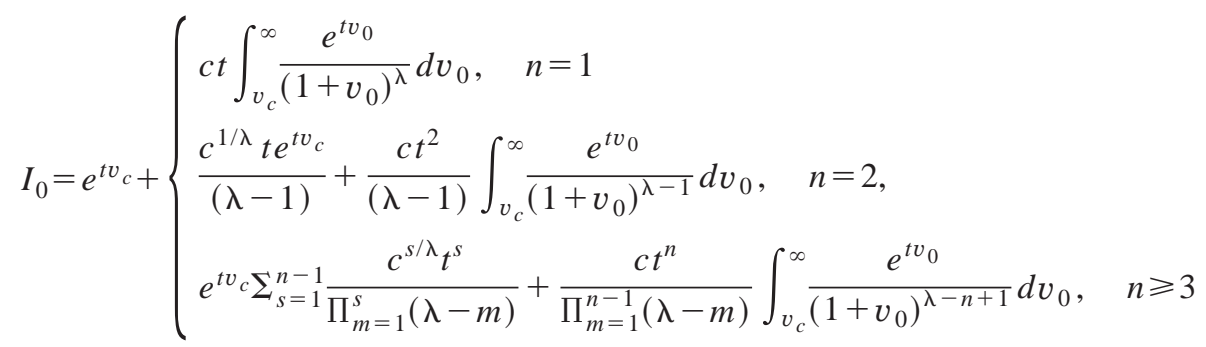

where $t=-q \ln 2$. We must now tackle the integral

$$
I_{2}=\int_{v_{c}}^{\infty} \frac{e^{t v_{0}}}{\left(1+v_{0}\right)^{\lambda-n+1}} d v_{0}
$$

appearing in the above expressions. For $\lambda=n$, letting $u=1$ $+v_{0}$, we have

$$
I_{2}=e^{-t}\left\{E_{1}(-t)-\int_{1}^{1+v_{c}} \frac{e^{t u}}{u} d u\right\}
$$

where $E_{1}(z)=\int_{1}^{\infty}\left(e^{-z x} / x\right) d x$ is the exponential integral. Using the identity

$$
E_{1}(z)=-\gamma-\ln z-\sum_{n=1}^{\infty} \frac{(-1)^{n} z^{n}}{n n !}
$$

valid for $|z|<\pi$ ( $\gamma$ is Euler's constant) and expanding out the exponential, we obtain for $\lambda=n$ and $0<|t| \ll 1$

$$
I_{2} \approx-\left\{\gamma+\ln \left(1+v_{c}\right)\right\}-\ln (-t) .
$$

If $\lambda \neq n$ we estimate $I_{2}$ by making the change of variable $x=\left(-t\left(1+v_{0}\right)\right)^{n-\lambda}$ and again for $0<|t| \ll 1$ we obtain

$$
I_{2} \underset{t \rightarrow 0}{\approx} I_{3} \frac{(-t)^{\lambda-n}}{(n-\lambda)}-\frac{\left(1+v_{c}\right)^{n-\lambda}}{(n-\lambda)}+\cdots
$$

where $I_{3}=\int_{0}^{\infty} e^{-x^{1 /(n-\lambda)}} d x$ is a finite quantity. Putting the results for $I_{2}$ into Eq. (A3) and letting $t \rightarrow 0$ we obtain for $I_{0}$

$$
I_{0}=\left\{\begin{array}{l}
1-\frac{c I_{3}}{(1-\lambda)}(-t)^{\lambda}+\left\{\frac{\left(1+v_{c}\right)}{(1-\lambda)}-v_{c}\right\}(-t)+\cdots, \quad 0<\lambda<1 \\
1+\left\{c\left(\gamma+\ln \left(1+v_{c}\right)\right)-v_{c}\right\}(-t)+c(-t) \ln (-t)+\cdots, \quad \lambda=1 \\
1-\left\{v_{c}+\frac{\left(1+v_{c}\right)}{(\lambda-1)}\right\}(-t)+\frac{c I_{3}}{(\lambda-1)(2-\lambda)}(-t)^{\lambda}+\left\{\frac{v_{c}^{2}}{2}+\frac{v_{c}\left(1+v_{c}\right)}{(\lambda-1)}-\frac{\left(1+v_{c}\right)^{2}}{(\lambda-1)(2-\lambda)}\right\}(-t)^{2}+\cdots, \quad 1<\lambda<2 \\
1-\left\{v_{c}+\frac{\left(1+v_{c}\right)}{(\lambda-1)}\right\}(-t)-\frac{c}{(\lambda-1)}(-t)^{2} \ln (-t)+\left\{\frac{v_{c}^{2}}{2}+\frac{v_{c}\left(1+v_{c}\right)}{(\lambda-1)}-\frac{c\left(\gamma+\ln \left(1+v_{c}\right)\right)}{(\lambda-1)}\right\}(-t)^{2}+\cdots, \quad \lambda=2 \\
1+\sum_{s=1}^{n}(-1)^{s}\left\{\frac{v_{c}^{s}}{s !}+\sum_{w=0}^{s-1} \frac{v_{c}^{w}\left(1+v_{c}\right)^{s-w}}{w ! \Pi_{m=1}^{s-w}(\lambda-m)}\right\}(-t)^{s}+\frac{(-1)^{n} c}{(n-\lambda) \Pi_{m=1}^{n-1}(\lambda-m)}\left\{I_{3}(-t)^{\lambda}-\left(1+v_{c}\right)^{n-\lambda}(-t)^{n}\right\}+\cdots, \quad n-1<\lambda<n, n \geqslant 3 \\
1+\sum_{s=1}^{n}(-1)^{s}\left\{\frac{v_{c}^{s}}{s !}+\sum_{w=0}^{s-1} \frac{v_{c}^{w}\left(1+v_{c}\right)^{s-w}}{w ! \Pi_{m=1}^{s-w}(\lambda-m)}\right\}(-t)^{s}-\frac{(-1)^{n} c}{\Pi_{m=1}^{n-1}(\lambda-m)}\left\{(-t)^{n} \ln (-t)+\left(\gamma+\ln \left(1+v_{c}\right)\right)(-t)^{n}\right\}+\cdots, \quad \lambda=n, n \geqslant 3 .
\end{array}\right.
$$

Now we must evaluate the integral

$$
I_{1}=\int_{0}^{\infty}\left(1-e^{-v_{0} \ln 2}\right)^{q} \widetilde{\rho}\left(v_{0}\right) d v_{0} .
$$

Via a binomial expansion we have that

$$
I_{1}=1+\sum_{n=1}^{\infty}\left\{\frac{(-1)^{n}}{n !} \prod_{m=0}^{n-1}(q-m) \int_{0}^{\infty} e^{-n v_{0} \ln 2} \widetilde{\rho}\left(v_{0}\right) d v_{0}\right\}
$$

and collecting together the first and second powers of $q$

$$
\begin{aligned}
I_{1}= & -\left\{\int_{0}^{\infty} \sum_{n=1}^{\infty} \frac{1}{n} e^{-n v_{0} \ln 2} \widetilde{\rho}\left(v_{0}\right) d v_{0}\right\} q \\
& +\left\{\int_{0}^{\infty} \sum_{n=2}^{\infty} \frac{H(n)}{n !} e^{-n v_{0} \ln 2} \widetilde{\rho}\left(v_{0}\right) d v_{0}\right\} q^{2}+\cdots \\
= & +\left\{\int_{0}^{\infty} \ln \left(1-e^{-v_{0} \ln 2}\right) \widetilde{\rho}\left(v_{0}\right) d v_{0}\right\} q \\
& +\left\{\int_{0}^{\infty} \sum_{n=2}^{\infty} \frac{H(n)}{n !} e^{-n v_{0} \ln 2} \widetilde{\rho}\left(v_{0}\right) d v_{0}\right\} q^{2}+\cdots,
\end{aligned}
$$

where 


$$
H(n)=\left\{\begin{array}{l}
1, \quad n=2 \\
3, \quad n=3 \\
(n-3) !(2 n-3)+(n-1) ! \sum_{m=1}^{n-3} \frac{1}{m}, \quad n \geqslant 4 .
\end{array}\right.
$$

Putting the results for $I_{0}$ and $I_{1}$ into Eq. (A2) and taking out a factor of 2 we have

$$
E\left[\mathbf{M}_{0}^{q}+\left(1-\mathbf{M}_{0}\right)^{q}\right]=2\left[1+\frac{\left(I_{0}+I_{1}-2\right)}{2}\right] .
$$

Substituting into Eq. (A1) and noting that all the terms in $\left(I_{0}+I_{1}-2\right)$ go to zero as $q \rightarrow 0$, we have that for $0<q \ll 1$

$$
\tau_{A}(q) \simeq-1-\frac{\left(I_{0}+I_{1}-2\right)}{2 \ln 2} .
$$

Recalling that $(-t)=q \ln 2$ and keeping only the leading terms we obtain for $0<q \ll 1$

$$
\tau_{A}(q) \simeq-1+\left\{\begin{array}{l}
c_{1} q-c_{5} q^{2}+\cdots, \quad \lambda>2 \\
c_{1} q+c_{3} q^{2} \ln q+\cdots, \quad \lambda=2 \\
c_{1} q-c_{6} q^{\lambda}+\cdots, \quad 1<\lambda<2 \\
-c_{2} q \ln q-c_{7} q+\cdots, \quad \lambda=1 \\
c_{4} q^{\lambda}+\cdots, \quad 0<\lambda<1,
\end{array}\right.
$$

where $c_{1}, c_{2}, \ldots$ are functions of $\lambda$. Of particular note is

$$
c_{1}=\frac{1+\lambda v_{c}}{2(\lambda-1)}-\frac{1}{2} \int_{0}^{\infty} \log _{2}\left(1-e^{-v_{0} \ln 2}\right) \widetilde{\rho}\left(v_{0}\right) d v_{0}=\alpha_{0}(\lambda) .
$$

Applying the Legendre transformation

$$
f(\alpha)=\alpha q-\tau(q)
$$

$$
\alpha=\frac{d \tau(q)}{d q}
$$

we obtain for $f(\alpha)$

$$
f(\alpha) \simeq 1-\left\{\begin{array}{l}
b_{1}\left[\alpha_{0}(\lambda)-\alpha\right]^{\gamma}, \quad \lambda>1, \alpha \nearrow \alpha_{0}(\lambda) \\
b_{2} e^{-c^{\prime} \alpha}, \quad \lambda=1, \alpha \rightarrow \infty \\
b_{3} \alpha^{\kappa}, \quad 0<\lambda<1, \alpha \rightarrow \infty
\end{array}\right.
$$

where $b_{1}, b_{2}$, and $b_{3}$ are positive constants for a fixed $\lambda$, $c^{\prime}=2 / c, \kappa=\lambda /(\lambda-1)$ and $\gamma=\max \{\lambda /(\lambda-1), 2\}$.
[1] T. C. Halsey, M. H. Jensen, L. P. Kadanoff, I. Procaccia, and B. I. Shraiman, Phys. Rev. A 33, 1141 (1986).

[2] H. G. E. Hentschel and I. Procaccia, Physica D 8, 435 (1983).

[3] R. Benzi, G. Paladin, G. Parisi, and A. Vulpiani, J. Phys. A 17, 3521 (1984).

[4] U. Frisch and G. Parisi, in Turbulence and Predictability of Geophysical Flows and Climate Dynamics, edited by M. Ghil, R. Benzi, and G. Parisi (North-Holland, New York, 1985).

[5] B. B. Mandelbrot, J. Fluid Mech. 62, 331 (1974).

[6] C. J. G. Evertsz and B. B. Mandelbrot, in Chaos and Fractals: New Frontiers of Science, edited by H.-O. Peitgen, H. Jurgens, and D. Saupe (Springer-Verlag, New York, 1992).

[7] C. Meneveau and K. R. Sreenivasan, Phys. Rev. Lett. 59, 1424 (1987).

[8] H. G. E. Hentschel, Phys. Rev. E 50, 243 (1994).
[9] Y. Hayakawa, S. Sato, and M. Matsushita, Phys. Rev. A 36, 1963 (1987).

[10] Ph. Brax and R. Peschanski, Nucl. Phys. B 346, 65 (1990).

[11] B. B. Mandelbrot, C. J. G. Evertsz, and Y. Hayakawa, Phys. Rev. A 42, 4528 (1990).

[12] J. Lee and H. E. Stanley, Phys. Rev. Lett. 61, 2945 (1988).

[13] R. Blumenfeld and A. Aharony, Phys. Rev. Lett. 62, 2977 (1989).

[14] A. Csordás and P. Szépfalusy, Phys. Rev. A 39, 4767 (1989).

[15] W. H. Press, S. A. Teukolsky, W. T. Vetterling, and B. P. Flannery, Numerical Recipes in C (Cambridge University Press, Cambridge, England, 1992).

[16] B. B. Mandelbrot and C. J. G. Evertsz, Nature (London) 348, 143 (1990). 